\title{
Potential of Pembrolizumab in Metastatic or Recurrent Head and Neck Cancer: Evidence to Date
}

This article was published in the following Dove Press journal: OncoTargets and Therapy

\author{
Michael G McCusker (ID \\ Dennis Orkoulas-Razis ${ }^{2}$ \\ Ranee Mehra (iD) \\ 'University of Maryland Medical Center, \\ Greenebaum Comprehensive Cancer \\ Center, Baltimore, MD, USA; \\ ${ }^{2}$ Department of Internal Medicine, \\ University of Maryland Medical Center, \\ Baltimore, MD, USA
}

\begin{abstract}
Relapsed and/or metastatic head and neck squamous cell carcinoma (R/M HNSCC) is a heterogeneous disease previously associated with poor prognosis and limited treatment options until the advent and implementation of immune checkpoint inhibitors (ICIs). The fully humanized monoclonal antibody pembrolizumab alone, or in combination with chemotherapy, was shown to have significantly improved overall survival (OS) when compared to the standard of care (SOC) EXTREME regimen consisting of the monoclonal antibody cetuximab combined with a platinum and 5-fluorouracil. Pembrolizumab with or without chemotherapy will soon supplant the EXTREME regimen that has been in use for over a decade. Given the fast-approaching significant change in the treatment algorithm for $\mathrm{R} / \mathrm{M}$ HNSCC and the novelty of ICIs in general, it is important to review the literature to date to understand how this rapidly growing treatment class has come about and explore potential areas of research for the plethora of questions that remain unanswered in selecting patients appropriate for treatment with ICIs in the R/M setting. In this review, we explore the landmark trials leading to the use of ICIs for R/M HNSCC with a particular focus on pembrolizumab, the most well-studied ICI in this setting. We also provide an overview of the rationale behind the use of ICIs in relation to the immune system and challenges surrounding tumor heterogeneity and PD-L1 expression status, human papilloma virus (HPV) and the efficacy of ICI, potential of radiation therapy for enhancement of ICI response, and complications of immune-related adverse events (irAEs).
\end{abstract}

Keywords: head and neck cancer, immunotherapy, pembrolizumab, checkpoint inhibitors

\section{Introduction}

Head and neck cancer is the sixth most common malignancy worldwide with approximately 600,000 new cases diagnosed each year. ${ }^{1}$ A heterogeneous group of tumors arising from squamous epithelium of the lips, oral cavity, oropharynx, and larynx accounts for $90-95 \%$ of head and neck cancers. Multimodal therapies with surgery, radiation, and/or platinum-based chemoradiation are the mainstay of treatment for locally advanced (LA) head and neck squamous cell carcinoma (HNSCC), yet disease recurs frequently $(60 \%){ }^{2}$ Patients with either locoregionally recurrent or metastatic $(30 \%)$ disease are left with few treatment options and poor prognoses. ${ }^{3,4}$

For relapse and/or metastatic (R/M) HNSCC not amenable to curative-intent treatment, first-line palliative chemotherapy was the mainstay of treatment for decades despite poor overall survival (median OS 10 months) and high morbidity. The "EXTREME" regimen consisting of the monoclonal antibody cetuximab which targets the epidermal growth factor receptor (EGFR), combined with a platinum and 5-fluorouracil was a commonly utilized regimen for fit patients. ${ }^{5}$ After progression,
Correspondence: Ranee Mehra University of Maryland Medical Center, Greenebaum Comprehensive Cancer Center, 22 S. Greene St, Baltimore, MD 2I20I, USA

Tel + I 4I0-328-7904

Fax + I 410-328-0128

Email ranee.mehra@umm.edu 
treatment was usually limited to single-agent therapies; methotrexate was most commonly used and demonstrated an overall response rate (ORR) of approximately $6 \%$ and a median OS of 6 months. ${ }^{6}$ Treatment options for $\mathrm{R} / \mathrm{M}$ disease that progressed beyond single-agent chemotherapy were severely limited until the development and implementation of a new class of immunotherapy in 2016.

PD-1 inhibitors are approved for the treatment of patients with R/M HNSCC who progressed on or after platinum-based chemotherapy. Monoclonal antibodies against programmed death receptor-1 (PD-1), programmed death receptor ligand-1 (PD-L1), and cytotoxic T-lymphocyte-associated protein 4 (CTLA-4) have shown lasting responses in a number of cancers and were rapidly expanded to use in HNSCC. To date, several ICIs have been studied in HNSCC (Table 1) and two of the most well-studied ICIs for the treatment of HNSCC in the R/M setting are the PD-1 inhibitors pembrolizumab and nivolumab. These agents were granted accelerated approval by the United States Food and Drug Administration (FDA) based on early data from the nonrandomized KEYNOTE012 trial $^{7}$ for pembrolizumab and the CHECKMATE- $141^{8}$ trial for nivolumab. While there have been promising results with ICIs, optimal patient selection for treatment with ICIs has remained elusive. ${ }^{9}$ Fortunately, a number of investigations have addressed important patient- and disease-associated factors to guide clinicians in appropriate patient selection. This review examines available data on ICIs and, in particular, updated data with pembrolizumab for use in the treatment of HNSCC as it relates to PD-L1 expression, human papilloma virus (HPV) infection, radiation therapy, and immune-related adverse events (irAEs).

\section{Biology of Head and Neck Squamous Cell Carcinoma}

Genetic alterations and immune system disruption are the hallmark of cancer and HNSCC is a highly immunogenic cancer. Infection with high-risk HPV is a contributor to the pathogenesis of HNSCC and oropharyngeal cancer in particular in a large subset of HNSCC cancers, and the incidence of HPV-associated HNSCC continues to rise. $\mathrm{HPV}$-associated HNSCC is found in approximately $40-80 \%$ of patients in the United States, ${ }^{3}$ as opposed to Europe where tobacco- and alcohol-associated HNSCC is more prevalent than HPV-related disease. Overall, HPVassociated HNSCC of oropharyngeal origin is seen in younger, predominantly white males of higher socioeconomic status (SES). ${ }^{10,11}$ Genotypes 16 and 18 are more commonly seen in the United States compared to Europe and Asia, but this is largely due to the fact that HNSCC is primarily non-HPV related in these regions. ${ }^{12} \mathrm{HPV}$ genotype 16 (p16) accounts for over $90 \%$ of cases. ${ }^{13}$ HNSCC was down-trending in the 1980s, when alcohol and tobacco were the primary drivers of carcinogenesis. However, a rise in HPV infection has correlated with an increased incidence of HNSCC. ${ }^{14}$ This is reflected in a recent incidence analysis per 100,000 of HPV-positive (4.62, 95\% CI=4.51-4.73) versus HPV-negative (1.82, $95 \% \mathrm{CI}=1.75-1.89)$ HNSCC patients. ${ }^{11}$

In HPV-associated disease, HPV promotes carcinogenesis by integrating into the DNA of the host leading to activation of oncoproteins E6 and E7, which in turn dysregulate tumor suppressors TP53 and Rb, respectively. TP53, one of the most important tumor suppressor genes encoding for a transcription factor with roles in DNA repair, cell cycle regulation, apoptosis, and genomic stability is mutated in approximately $80 \%$ of HPV-negative cases, as TP53 is degraded by HPV oncoprotein E6 in HPV-positive disease. $^{15,16}$ The aforementioned process leads to immune response evasion through cytokine and chemokine expression and antigen presentation, IFN $\gamma$ pathway down-regulation, and an immune-privileged state for the tumor. ${ }^{17}$ In contrast, HPVnegative HNSCC is associated with TP53 mutations and down-regulation of $\mathrm{p} 16 .^{3}$ Thus, $\mathrm{p} 16$ is a biomarker for HPV

Table I Immunotherapies and PD-LI Testing Methods

\begin{tabular}{|c|c|c|c|c|c|c|}
\hline Agent & Class & Target & PD-LI Antibody & Antibody Host Species & Platform 82 & PD-LI Positivity Cut-Off \\
\hline Pembrolizumab & $\lg G 4$ & PD-I & $22 \mathrm{C} 3 *$ & Mouse & Dako Autostainer Link 48 & TC or IC $>1 \%,>50 \%$ \\
\hline Nivolumab & $\lg G 4$ & PD-I & $28-8 *$ & Rabbit & Dako Autostainer Link 48 & TC $>1 \%,>5 \%,>10 \%$ \\
\hline Atezolizumab & $\lg G \mid$ & PD-LI & $\mathrm{SPI} 42^{\dagger}$ & Rabbit & Ventana Benchmark Ultra & $\mathrm{TC} \geq 5 \% ; \mathrm{IC} \geq 5 \%$ \\
\hline Durvalumab & $\lg G \mid$ & PD-LI & $\mathrm{SP} 263^{\dagger}$ & Rabbit & Ventana Benchmark Ultra & $\mathrm{TC} \geq 25 \%$ \\
\hline Avelumab & $\operatorname{lgG} \mid$ & PD-LI & $73-10 *$ & Rabbit & Dako Autostainer Link 48 & N/A \\
\hline
\end{tabular}

Notes: *Dako North America. †Ventana Medical Systems Inc., Tucson, AZ.

Abbreviations: TC, tumor cells; IC, immune cells. 
infection amongst oropharyngeal primary sites, with a cutoff point greater than $70 \%$ by immunohistochemistry (IHC) considered HPV-positive disease. ${ }^{18}$ TP53 mutations occur early in carcinogenesis and are associated with poor prognosis, therapy resistance, and increased recurrence rate in both HPVpositive and negative disease. ${ }^{19}$ The retinoblastoma (RB) tumor suppressor regulates cell cycle progression and also represents an early alteration in HNSCC tumorigenesis. In HPV-positive disease, viral protein E7 degrades RB and leads to E2F activation and uncontrolled proliferation. ${ }^{20,21}$ Further mutations have been discovered, ${ }^{22}$ beyond those previously identified that are involved in cell cycle regulation, apoptosis and DNA repair, and mutations in genes involved in the regulation of squamous cell differentiation (eg NOTCH1, ${ }^{23}$ IRF6, ${ }^{24}$ and $\mathrm{TP}^{25}{ }^{25}$ ). Important differences in the specific alterations in PI3K mutations between HPV-associated and HPV-unassociated disease have also been noted and may provide inferences on clinical outcomes for treatment with PI3K/mTOR inhibitors. ${ }^{26,27}$ These known and yet-to-bediscovered genomic alterations as well as familial susceptibility (e.g. Fanconi anemia ${ }^{28}$ ) to HSNCC will continue to clarify and identify potential therapeutic targets.

\section{Clinical Trials with Immune Checkpoint Inhibitors}

The Phase Ib KEYNOTE-012 trial was one of the first studies to evaluate ICI in a population of patients with $\mathrm{R} /$ M HNSCC. Patients who had previously failed treatment with curative intent were enrolled. Sixty-three percent of patients had received prior platinum and cetuximab therapy. Historically, the expected response rate (RR) of thirdline treatment in this setting was in the single digits. ${ }^{29}$ The safety and efficacy of pembrolizumab in R/M HNSCC was evaluated across two cohorts. The initial cohort required PD-L1 expression of $1 \%$ by IHC. Patients $(\mathrm{N}=60)$ were treated at a dose of $10 \mathrm{mg} / \mathrm{kg}$ every 2 weeks. An expansion cohort $^{30}$ was treated at a fixed dose of $200 \mathrm{mg}(\mathrm{N}=132)$. In the initial report, the overall response rate (ORR) was $18 \%$ (95\% CI=8-32). Median progression-free survival (PFS) was 2 months $(95 \% \mathrm{CI}=2-4)$. Drug-related adverse events of any grade were $63 \%(n=38)$, with $17 \%(n=10)$ grade 3 and zero grade 4 drug-related adverse events, respectively. ${ }^{7}$ These promising results lead to the accelerated approval of pembrolizumab for R/M HNSCC by the FDA on August 6, 2016, ${ }^{31}$ with future studies confirming the efficacy of pembrolizumab in the $\mathrm{R} / \mathrm{M}$ setting.
In the pooled analysis $(\mathrm{N}=192)$ of the initial $(\mathrm{n}=60)$ and expansion $(n=132)$ cohorts of KEYNOTE-012, Mehra et al confirmed the efficacy and safety of pembrolizumab for R/ M HNSCC. ${ }^{32}$ In the combined cohort, significantly higher response rates were observed in patients with (21\%) versus without (6\%) PD-L1 expression using combined positive score (CPS, $p=0.023)^{33}$ but not in patients with $(18 \%)$ versus without (19\%) PD-L1 expression by tumor proportion score (TPS, $p=0.461$ ). Similar differences were observed for both PFS and OS when PD-L1 expression was assessed using CPS or TPS as outlined in the pooled analysis supplemental tables. The analysis also showed a correlation between PD-L1 and PD-L2 expression ( $\mathrm{p}<0.001)$ with a higher ORR seen in patients expressing both PD-L1 and PD-L2 (23\%) versus those only expressing PD-L1 (10\%). Still, patients without expression of either biomarker responded at a clinical response rate of 9\% suggesting that PD-L1 and PD-L2 biomarkers do not fully predict response to pembrolizumab, highlighting the need for further studies addressing novel predictive molecular signatures. Clearly, subsets of patients are potentially curable as $4 \%$ of patients in the pooled analysis obtained a complete response (CR) with pembrolizumab.

Noting the importance of the tumor microenvironment (TME) in ICI efficacy, the Phase II single-arm KEYNOTE-055 $5^{34}$ study of 171 patients with R/M HNSCC further evaluated the exploratory biomarker CPS on patient outcomes given the response to pembrolizumab observed in the KEYNOTE- $012^{7}$ trial. Patients resistant to both platinum chemotherapy and cetuximab with progression or recurrence within 6 months of the last dose of therapy were treated with pembrolizumab $200 \mathrm{mg}$ IV every 3 weeks until disease progression or intolerance. The overall response rate for all patients was 16\% (95\% $\mathrm{CI}=11-23$ ) with adverse events of any grade reported in $64 \%$ of patients $(n=109)$ and grade 3 or greater in $15 \%$ of patients $(n=26)$. In a retrospective analysis, based on a CPS cut-off of $\geq 1 \%$, ORR was $18 \%(95 \% \mathrm{CI}=12-25)$ in CPS positive patients and $12 \%(95 \% \mathrm{CI}=2-30)$ in $\mathrm{CPS}$ negative patients. With a higher cutoff of CPS $\geq 50 \%$, ORR dichotomies were even larger, changing to $27 \%$ $(95 \% \mathrm{CI}=15-42)$ and $13 \%(95 \% \mathrm{CI}=7-20)$ in $\mathrm{CPS}$ positive and negative patients, respectively. Similar to KEYNOTE$012{ }^{7}$ the KEYNOTE- $055^{34}$ trial does not advocate for the use of CPS as a determinate of patient selection, as a percentage of CPS negative patients still benefited from pembrolizumab, but only notes that PD-L1 expression is associated with improved outcomes. 
The role of pembrolizumab in the treatment of $\mathrm{R} / \mathrm{M}$ HNSCC was further studied in the randomized, open-label, international, Phase III KEYNOTE-040 study. ${ }^{9}$ Expanding upon the results of the single-arm KEYNOTE- $012^{7}$ and KEYNOTE- $055^{34}$ trials, 495 patients were randomly assigned to receive pembrolizumab $(n=247)$ or investigator's choice $(n=248)$ of methotrexate, docetaxel, or cetuximab. At the time of prespecified final analysis of death, the outcome measure was lower in patients treated with pembrolizumab $(72 \%)$ than those treated with SOC $(80 \%, \mathrm{HR}=0.82$, $p=0.0316$ ) though the HR did not meet the predefined boundary for efficacy. However, after confirming the survival status of 12 patients, pembrolizumab met the efficacy boundary for OS with a one-sided alpha of less than 0.0175. Median OS was 8.4 months with pembrolizumab and 6.9 months with SOC, respectively. The benefit of pembrolizumab versus SOC was greater in patients with PD-L1 expression in both the tumor and the TME. The KEYNOTE- $040^{9}$ trial showed the benefits of pembrolizumab over single-agent chemotherapy, a common third-line option for patients with R/M HNSCC.

In a trial investigating the PD-1 inhibitor nivolumab in recurrent HNSCC, the CHECKMATE- $141^{8}$ trial addressed the same question as to the KEYNOTE- $040^{9}$ trial. The phase III trial randomized 361 patients to receive nivolumab $(n=240)$ or standard therapy $(n=121)$ of methotrexate, docetaxel, or cetuximab as in KEYNOTE-040. Similarly, the primary endpoint was OS with secondary endpoints being PFS and the rate of objective response. Median OS for nivolumab was 7.5 months versus 5.1 months with SOC and median PFS was 2.0 months versus 2.3 months, respectively. However, there was a late separation in the KaplanMeyer curve, with PFS at 6 months of $19.7 \%$ with nivolumab versus $9.9 \%$ with SOC, respectively. These figures mirror KEYNOTE-040, albeit with nivolumab demonstrating shorter OS and PFS.

The important question of whether PD-1 inhibitor treatment is superior to the EXTREME regimen in R/M HNSCC was addressed in the KEYNOTE- $048^{35}$ trial. For over a decade, the EXTREME regimen ${ }^{5}$ has been the SOC firstline treatment for R/M HNSCC. The randomized, openlabel, international, phase III trial compared pembrolizumab monotherapy ( $\mathrm{n}=301)$ to the EXTREME regimen $(\mathrm{n}=300)$ or pembrolizumab plus chemotherapy $(\mathrm{n}=126$, cisplatin or carboplatin plus 5-fluorouracil) to EXTREME $(n=110)$. The study analyzed two lower boundaries for maximum CPS positivity of $\geq 20$ and $\geq 1$. At the final analysis, superior OS was seen in patients with PD-L1 CPS $\geq 20$, CPS $\geq 1$, and the total population for pembrolizumab plus chemotherapy versus EXTREME. When pembrolizumab monotherapy was compared to EXTREME, OS was superior for patients with CPS $\geq 20$ and $\geq 1$, but not the overall population. Treatment-related adverse events were similar to previous trials. These data support the use of either pembrolizumab plus platinum-based chemotherapy in patients with $\mathrm{R} / \mathrm{M}$ HNSCC, or pembrolizumab monotherapy for use in patients PD-L1 positive tumors as a new first-line treatment for R/M HNSCC. Future studies should further define appropriate patient selection for ICI treatment as current hypotheses remain largely theoretical and preclinical, and evidence of biomarkers for predicting response to therapy is lacking. Still, PD-L1 expression is the most widely used and heavily studied biomarkers for response to ICI, but clearly does not fully predict response to ICI.

\section{Predictors of Response to Immune Checkpoint Inhibitors PD-LI Expression}

The human immune system is a tightly regulated, complex network of co-stimulatory and co-inhibitory signals that serve as "immune checkpoints" to prevent exaggerated immune response and autoimmunity. $\mathrm{T}$ cell activation requires antigen recognition through human leukocyte antigen (HLA) peptide presentation combined with a "second signal" for full activation that is balanced by coinhibitory signals. ${ }^{36}$ Tumors may exploit this balance through overexpression of "self" antigens to evade immune activation and destruction, and promote tumor proliferation, angiogenesis, and metastasis. ${ }^{37}$ Several checkpoints for potential tumor escape have been described, such as CTLA-4, $\mathrm{T}$ cell immunoglobulin mucin domain-3 (TIM-3), lymphocyte-activation gene 3 (LAG-3), and PD-1/PD-L1. ${ }^{38-40}$ PD-1 (CD279) is a CD28 family transmembrane receptor expressed on the surface of activated T cells, B cells, natural killer (NK) cells, and monocytes, but is absent on naïve $\mathrm{T}$ cells. ${ }^{41}$ Binding of PD-1 to its major ligands PD-L1 and PD-L2 causes the release of inhibitory cytokines and a "checked" $\mathrm{T}$ cell response. ${ }^{42}$ Pembrolizumab is a fully humanized IgG4 monoclonal antibody against PD-1, which seeks to disrupt this interaction and restore balance to allow for immune system recognition, activation, and destruction of tumor cells. ${ }^{43}$ In HNSCC, PD-L1 overexpression is found in $50-60 \%$ of patients, ${ }^{44}$ making it an appealing target for immunotherapy in patients with $\mathrm{R} / \mathrm{M}$ disease. In general, PD-L1 expressing tumors tend to show improved response 
to anti-PD-1-/anti-PD-L1 therapies in comparison to PDL1 non-expressing tumors across an array of different tumor types. ${ }^{45-47}$ Based on this rationale, a series of clinical trials have been conducted to study pembrolizumab (Table 2) in addition to other ICIs (Table 3) for HNSCC.

PD-L1 expression is highly variable and its predictive value in HNSCC is partially influenced by three major factors: (1) Incongruent use of PD-L1 assays between laboratories, (2) varying levels of PD-L1 positivity cutoff, and (3) intra-tumoral heterogeneity. Studies evaluating PD-L1 expression employ different protocols, antibodies, scoring systems, and cutoffs for positivity. The landmark randomized, open-label, phase III CHECKMATE- $141^{8}$ trial randomized 361 patients with recurrent HNSCC to nivolumab versus $\mathrm{SOC}$ in a 2:1 ratio. PD-L1 expression was determined using a rabbit antihuman PD-L1 antibody by immunohistochemical (IHC) testing (Dako North America, clone 28-8, Epitomics) using cut-off levels of $1 \%$ or more, $5 \%$ or more, and $10 \%$ or more in a minimum of 100 tumor cells. In contrast, the phase III KEYNOTE$048^{48}$ trial evaluated PD-L1 status using PD-L1 22C3 pharmDx assay (Agilent) and was scored using TPS of $50 \%$ or more and CPS of 20 or more. PD-L1 protein expression determined by TPS is defined as the percentage of viable tumor cells showing partial or complete membrane staining. A combined positive score represents the number of PD-L1 staining cells (tumor cells, lymphocytes, and macrophages) divided by the total number of viable tumor cells and is presented as a percentage. In the international, multi-institutional, phase II HAWK study of durvalumab for patients with R/M HNSCC, the VENTANA PD-L1 (SP263) Assay (Ventana Medical Systems, Inc., Tucson, AZ, USA) was used and PD-L1 tumor cells were scored PD-L1 high if expression was $25 \%$ or more; ${ }^{49}$ OS was 7.1 months $(95 \% \mathrm{CI}=4.9-9.9)$ in patients treated with durvalumab, a human immunoglobulin G1 kappa (IgG1 $\kappa)$ monoclonal antibody that blocks the interaction of PD-L1 with PD-1. In general, comparisons across trials are not advised, but as more immunotherapybased treatments and combination therapies are developed, a standardized approach is needed to ensure proper determination of PD-L1 as a predictive marker of response to ICI.

Intra-tumoral heterogeneity challenges pathologists across a number of tumor types, and HNSCC is no exception. In a study of intra-tumoral PD-L1 expression variability, Rasmussen et $\mathrm{al}^{50}$ performed 6 random core biopsies on 33 surgical specimens from 28 patients with
HNSCC and compared PD-L1 concordance percentages (all positive or all negative). With a cut-off of $1 \%$ or more PD-L1 expression, TPS and CPS concordance was $36 \%$ and $52 \%$, respectively. The concordance increased with a cut-off value of $50 \%$ or more for TPS (70\%) and CPS (54\%), respectively. Further, the negative predictive value (NPV) of a single negative biopsy with CPS using a cut-off value of $1 \%$ or more was $0 \%$ (ie none of the 6 biopsies from 33 samples had no PD-L1 expression). However, using the 50\% cut-off value, NPV was $79.9 \%$ with TPS and $62.8 \%$ with CPS. In a population of patients with R/M HNSCC where single biopsies for disease recurrence confirmation are common, the predictive value of PD-L1 expression using a single core biopsy gives caution to clinicians in determining if ICI is the appropriate treatment, and supports the necessity of tissue preservation at the time of surgery where PD-L1 expression may be assessed using multiple samples from both primary and metastatic disease sites.

\section{HPV Infection}

HPV-positive HNSCC is associated with a better overall prognosis in the setting of both locoregional and metastatic disease, in spite of a more aggressive disease course. In the ICON-S study, 5-year OS in HPV-positive HNSCC in stages I, II, III, IVa, and IVb were $88 \%, 82 \%, 84 \%, 81 \%$, and $60 \%$, respectively, versus 76\%, 68\%, 53\%, 45\%, and 34\% in HPVnegative HNSCC. ${ }^{10} \mathrm{HPV}$-positive tumors were found earlier (T1, T2) with more frequent nodal involvement. Still, HPVpositive tumors progress less frequently, and upon progression, a better OS is seen with a median OS of approximately 2.6 years and 0.8 years in HPV positive and negative tumors, respectively. $^{51}$ An analysis of HPV status in R/M HNSCC with SOC treatment showed a median OS in HPV-positive patients of 12.9 months versus 6.7 months in HPV-negative patients. Similar results were found in p16 positive (11.9 months) versus p16 negative (6.7 months) patients. ${ }^{52}$ A retrospective analysis of the EXTREME trial yielded comparable results. $^{53}$

The impaired ability of the immune system to control both HPV-positive and negative tumors is evidenced by high levels of cytotoxic CD8 positive and activated NK cell activity in HNSCC, yet diminished tumor control. The tumor microenvironment has been shown to suffer from defective tumor-infiltrating lymphocytes, impaired ability of T cells to recognize cancer cells, and activation of MAPK, STAT3, and B-catenin/wnt signaling pathways. ${ }^{54}$ Despite the heavy immune presence in the tumor microenvironment, tumors 


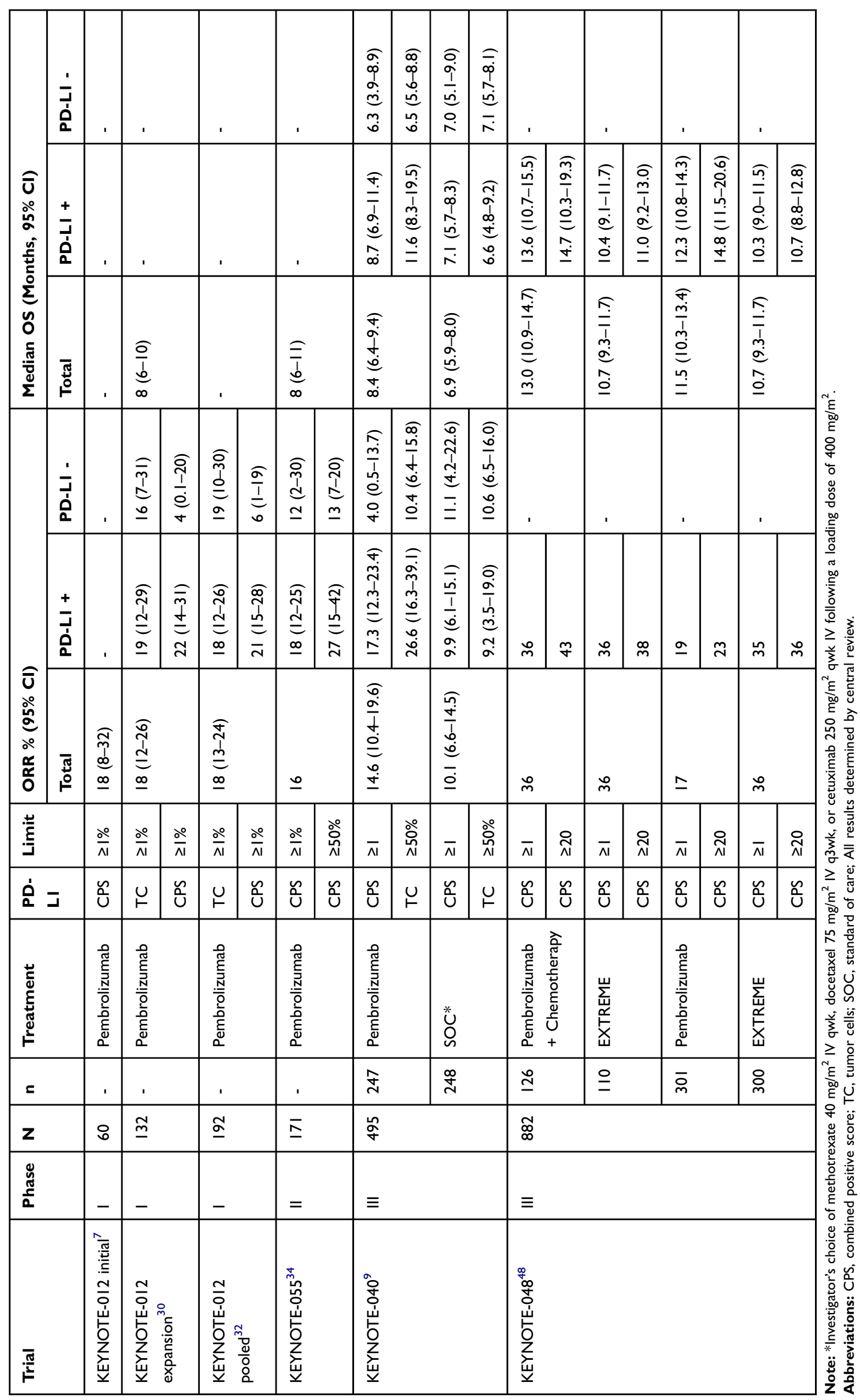




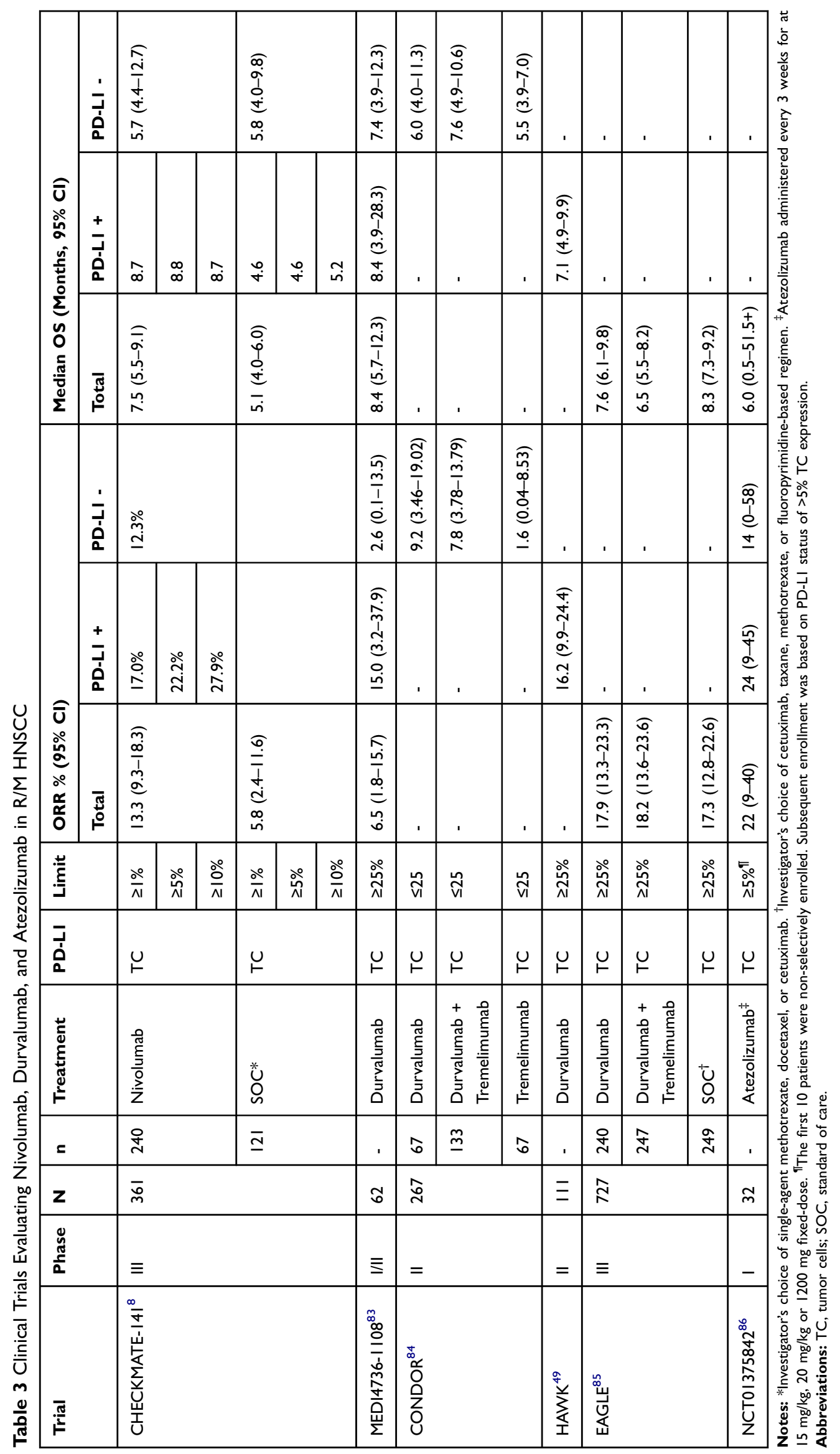


are able to escape control, leading to an important hypothesis of immune system "reprogramming" and exploitation of neoantigens on cancer cells as a result of genomic alterations. Entertainment of this hypothesis contributed to the development of ICIs that will soon become the first line for the treatment of R/M HNSCC and studies continue to clarify important biomarkers and genomic alterations that have predictive and prognostic value for response to ICI treatment.

Throughout the KEYNOTE trials, subset analyses were performed on the effect of HPV (p16) positivity and response to pembrolizumab. The phase $\mathrm{Ib}$ KEYNOTE- $012^{7}$ trial showed greater ORR and PFS in HPV-positive (25\%, 4 months) versus HPV-negative $(19 \%, 2$ months) tumors, respectively. The KEYNOTE-012 expansion cohort found similar differences in ORR in HPV-positive (32\%) and HPVnegative (14\%) tumors and 6 months PFS in HPV-positive (37\%) and HPV-negative (20\%) tumors. In the pooled data analysis of KEYNOTE- $012,{ }^{32}$ ORR was $24 \%$ and $16 \%$ in HPV-positive and HPV-negative tumors, respectively, with $18 \%$ demonstrating complete or partial response regardless of HPV status. Similar data were demonstrated in the phase II KEYNOTE- $055^{34}$ trial. However, PFS was similar among the two groups (2.1 months, $95 \% \mathrm{CI}=2.1-2.1)$ with a median 6-month OS of all patients being 59\% (72\% in HPV-positive versus $55 \%$ in HPV-negative). KEYNOTE- $048,{ }^{48}$ which compared pembrolizumab with or without chemotherapy to the EXTREME regimen, showed clinical benefit from pembrolizumab regardless of HPV status. Thus, although HPV status is associated with a better prognosis as demonstrated throughout the KEYNOTE trials, it should be considered independent of PD-L1 status, as PD-L1 expression is observed in HPV-negative HNSCC as well. This is also reflected in CHECKMATE- $141^{24}$ where 16 positive patients, regardless of PD-L1 status had improved OS with nivolumab (9.1 months), compared to SOC (4.1 months). In turn, OS in PDL1 positive patients was identical in the nivolumab group regardless of p16 status (8.8 months), underscoring that HPV status is a favorable independent prognostic factor, irrespective of treatment.

\section{Tumor Mutational Burden}

The genetic makeup and mutational burden of HNSCC has been extensively studied using microarray subgroup analyses based on mutational profile and predictive implications for response to immunotherapy are being defined. ${ }^{55}$ It is thought that tumors harboring more mutations may, in fact, lead to increased neoantigens and a more immunogenic response when treated with ICI. HPV-negative tumors (i.e., HNSCC likely secondary to risk factors such as tobacco and alcohol) generally have a higher TMB than HPV-positive tumors. ${ }^{56}$ In general, HNSCC tumors have high tumor mutational burden (TMB) when measured by a number of mutations per megabase ( $N$ mut/ $\mathrm{MB}$ ), as shown in a study using compressive genomic profiling of 1,184 HNSCC samples exhibiting a median of $5 \mathrm{mut} / \mathrm{MB}$, regardless of HPV status, ${ }^{57}$ and correlational studies of $\mathrm{TMB}^{58}$ and responsiveness to immunotherapy is an active area of research. ${ }^{59}$ In a pooled analysis evaluating the relationship between TMB and ORR for anti-PD-1 or anti-PD-L1 immunotherapies across multiple cancer types, Yarchoan et al observed a significant correlation between TMB and ORR with a correlation coefficient of $0.74(p<0.001)$. The analysis included 19 studies using nivolumab and 20 studies using pembrolizumab in HNSCC. Using data from four KEYNOTE clinical trials from more than 300 patient samples of 22 different tumor types, Cristescu et al assessed the individual and joint clinical utility of the predictive biomarkers of TMB and $\mathrm{T}$ cell-inflamed gene expression profile (GEP) on the best overall response (BOR) to ICI. Patients were grouped according to TMB high ( $\geq$ Youden Index cut points) versus TMB low ( $<$ Youden Index cut points) and GEP (high versus low). TMB and GEP were modestly correlated, and each was independently predictive of response to ICI across the KEYNOTE trials. In HNSCC BOR was greatest in TMB high/PD-L1 positive $(30 \%, 95 \% \mathrm{CI}=17.3-44.9)$ and lowest for TMB low/PD-L1 negative (9\%, 95\% $\mathrm{CI}=0.2-41.3)$, but not statistically significant. Similarly, BOR was greatest for TMB high/GEP high tumors (37\%, 95\% $\mathrm{CI}=21.8-54.0$ ) and lowest for TMB low/GEP low tumors $(0 \%, 95 \% \mathrm{CI}=0.0-21.8)$, but not statistically significant. ${ }^{60}$ In an analysis of biomarkers predictive of BOR to ICI in HNSCC, Seiwert et al evaluated a combined cohort of patients from KEYNOTE-012 $(n=261)$ and KEYNOTE-055 $(n=154)$. BOR was significantly correlated with TMB by whole-exome sequencing (WES), PD-L1 CPS, and GEP regardless of HPV status $(p<0.01)$. Responses were higher in patients with both high TMB and PD-L1 CPS or GEP than in patients with low TMB and high PD-L1 CPS or GEP. ${ }^{61}$ As seen, TMB, GEP, and PD-L1 expression are predictive of response to ICI. However, key factors remain unaddressed before TMB is to be fully incorporated into treatment algorithms, such as the interplay between HPV and TMB, and the role of chemo- and/or immune-radiotherapy in the induction of neoantigens and $\mathrm{T}$ cell activation. 


\section{Pembrolizumab and Radiation Therapy}

Radiation therapy is a mainstay of treatment for HNSCC and the effects of radiation on the immune system and responses to ICI are under active investigation with over 30 clinical trials in process for ICIs (e.g. NCT 03539198 , 03383094, 03085719, 03844763, 03313804, 03283605, and 03258554). Radiotherapy can lead to both direct toxicity and immunomodulatory responses, leading to tumor cell death via $\mathrm{T}$ cell activation. ${ }^{62}$ Irradiated, apoptotic tumor cells release antigens that prime and activate cytotoxic $\mathrm{T}$ cells, leading to an abscopal effect of primed $\mathrm{T}$ cells recognizing non-irradiated tumor tissue. ${ }^{63}$ Preclinical studies evaluating radiotherapy with PD-L1 blockade have shown promising results, with the leading hypothesis that immunotherapy disrupts tumor evasion of T cell-mediated death induced by radiotherapy. ${ }^{64}$ A retrospective analysis of KEYNOTE-01 in patients with NSCLC treated with pembrolizumab and radiotherapy showed significantly longer PFS (HR $=0.56,95 \% \quad \mathrm{CI}=0.34-0.9, p=0.019)$ and $\mathrm{OS}$ $(\mathrm{HR}=0.58,95 \% \mathrm{CI}=0.36-0.94, p=0.026) .{ }^{62}$ Improved outcomes have also been shown with metastatic lung cancer with brain metastases, whereby patients underwent stereotactic irradiation. ${ }^{65}$ Translation of the effects of radiation therapy on response to ICI and vice versa to HNSCC patients is not yet fully understood. For example, recent research has shown that resistance to radiotherapy plus PDL1 blockade may arise via the TIM-3 pathway and T reg activation. ${ }^{66}$

In HNSCC, the ability of pembrolizumab to interfere with the PD-1/PDL-1 interaction and allow for $\mathrm{T}$ cellmediated death of tumor cells primed by radiotherapy is promising for the future of HNSCC management. Early outcomes of ongoing Phase I and II trials (GORTEC $2015-01,{ }^{67}$ RTOG 3504, ${ }^{68}$ NCT02641093 ${ }^{69}$ ) have been encouraging, but some trials have demonstrated increased adverse effects, mostly Grade 1 and 2. There are currently multiple ongoing clinical trials addressing the relationship between ICI and radiotherapy ${ }^{70}$ and the move to ICI-based treatment to the front-line setting is under investigation. The JAVELIN Head and Neck 100 (NCT02952586) global, multicenter, randomized, double-blind, phase III trial is currently evaluating the use of the fully human $\operatorname{IgG} 1$ anti-PD-L1 antibody avelumab plus cisplatin-based CRT versus placebo plus CRT as first-line treatment for patients with LA-HNSCC..$^{71}$ A similar trial using pembrolizumab (NCT03040999), is the Phase III, randomized placebo- controlled, double-blind KEYNOTE-412 trial evaluating the efficacy and safety of pembrolizumab plus CRT in comparison to placebo plus CRT in the front-line setting for LA-HNSCC not treated by surgery. ${ }^{72}$ The open-label, phase III KEYNOTE-689 trial (NCT03765918) is evaluating the efficacy and safety of both neoadjuvant and adjuvant pembrolizumab in resectable LA-HNSCC. ${ }^{73}$

\section{Immune-Related Adverse Events with Pembrolizumab in Head and Neck Squamous Cell Carcinoma}

A developing understanding of irAEs as a consequence of ICI is still being defined, and best prevention and treatment strategies remain unclear. The cause of irAEs is thought to be related to the aberrant action of activated T cells. Several possible mechanisms behind irAEs include: 1) Exacerbation of subclinical inflammation by ICI, ${ }^{74}$ 2) crossreactivity of shared antigens between organs at risk and tumors, ${ }^{77-79}$ and 3) the negative impact of ICI on the gut microbiome. ${ }^{80,81}$ Early fears for clinicians using immunosuppression for treatment of irAEs were grounded in the fact irAEs were correlated with improvement in PFS, OS, and ORR, a phenomenon that was more notable in anti-PD -1/PD-L1 than anti-CTLA4 agents. ${ }^{74-76}$ Clinicians feared that dampening such a response would lead to inferior outcomes and prohibit the use of ICI in the future. However, as demonstrated in the KEYNOTE studies, irAEs are common, but are rarely severe enough to result in permanent discontinuation of ICI treatment.

In the KEYNOTE trials, no new adverse events using ICI were uncovered using pembrolizumab for HNSCC. At the final analysis of the phase III KEYNOTE-048 trial, adverse events of grades $3-5$ that occurred in $\geq 5$ participants occurred in pembrolizumab alone (55\%), pembrolizumab plus chemotherapy (85\%), and cetuximab with chemotherapy $(83 \%)$ that are in line with prior studies using pembrolizumab and alternative PD-1 and PD-L1 ICIs. Not surprisingly, the addition of chemotherapy was associated with an increased incidence of adverse events. A list of prespecified "adverse events of interest" was analyzed at final analysis and includes factors that today might be referred to as irAEs, such as hypothyroidism, pneumonitis, and severe skin reactions. Here, what can be thought of as irAEs of grades 3-5 occurred in pembrolizumab alone (7\%), pembrolizumab plus chemotherapy $(5 \%)$, and cetuximab plus chemotherapy (10\%). Hypothyroidism of any grade, the most common irAE, was more common in 
pembrolizumab alone (18\%) and pembrolizumab plus chemotherapy (16\%) than cetuximab plus chemotherapy $(6 \%)$. However, there were no instances of grades 3-5 hypothyroidism in any of the participants. Expectedly, chemotherapy containing regimens were associated with higher instances of blood and lymphatic system disorders of grade 3-5 with anemia, in particular, occurring in pembrolizumab $(5 \%)$, pembrolizumab plus chemotherapy (25\%), and cetuximab plus chemotherapy $(17 \%)$.

The risk of adverse events with higher intensity treatment regimens for R/M HNSCC has limited options for clinicians to treat patients with marginal performance status and heavily pretreated disease, but there is now a powerful single-agent option for patients who may not be candidates for combination chemoimmunotherapy. In contrast to the landmark phase III EXTREME trial, ${ }^{5}$ single agent immunotherapy was compared to chemoimmunotherapy in the KEYNOTE-048 trial. Outcomes were better, and the incidence of adverse events was lower with single-agent pembrolizumab than chemoimmunotherapy. ${ }^{35}$ Further, when comparing the chemoimmunotherapy groups, the pembrolizumab-containing group also outperformed the EXTREME regimen in terms of outcomes and adverse events, leaving clinicians with two options for treatment of R/M HNSCC: Less intensive singleagent immunotherapy with pembrolizumab and more intensive chemoimmunotherapy with pembrolizumab, cisplatin or carboplatin, and 5-fluorouracil. However, defining patient populations to benefit from ICI alone or chemoimmunotherapy will require further trials to identify predictive factors based on patient and tumor characteristics.

\section{Conclusions}

Immune checkpoint inhibitors have changed the landscape for the treatment of aggressive R/M HNSCC. Pembrolizumab with chemotherapy and pembrolizumab alone will soon become SOC in this setting. Still, there are many questions left unanswered in selecting patients and tumors that may respond favorably to ICIs. As more powerful and effective ICIs become available, further studies are needed on important topics, such as standardization for determination of PD-L1 expression between laboratories, clarification on ICI and its relation to timing and dosing with CRT, the role of combination CRT and ICI versus radiation therapy alone with ICI in the front-line setting, and the roles of molecular biomarkers such as PDL2, TMB, and genomic signatures on responses to ICI. Nevertheless, excitement abounds with the use of a new class of ICI with generally mild and well-tolerated side effects for a group of patients previously left with few and poor options for treatment of R/M HNSCC.

\section{Disclosure}

Dr Ranee Mehra reports research funds from Merck, Astra Zeneca, personal fees from Bayer, outside the submitted work. The authors report no other conflicts of interest in this work.

\section{References}

1. Bray F, Ferlay J, Soerjomataram I, Siegel RL, Torre LA, Jemal A. Global cancer statistics 2018: GLOBOCAN estimates of incidence and mortality worldwide for 36 cancers in 185 countries. CA Cancer J Clin. 2018;68(6):394-424. doi:10.3322/caac.v68.6

2. Chang JH, Wu CC, Yuan KS, Wu ATH, Wu SY. Locoregionally recurrent head and neck squamous cell carcinoma: incidence, survival, prognostic factors, and treatment outcomes. Oncotarget. 2017;8 (33):55600-55612. doi:10.18632/oncotarget.16340

3. Marur S, D'Souza G, Westra WH, Forastiere AA. HPV-associated head and neck cancer: a virus-related cancer epidemic. Lancet Oncol. 2010;11(8):781-789. doi:10.1016/S1470-2045(10)70017-6

4. Seiwert TY, Cohen EE. State-of-the-art management of locally advanced head and neck cancer. $B r \quad J$ Cancer. 2005;92 (8):1341-1348. doi:10.1038/sj.bjc.6602510

5. Vermorken JB, Mesia R, Rivera F, et al. Platinum-based chemotherapy plus cetuximab in head and neck cancer. $N$ Engl J Med. 2008;359 (11):1116-1127. doi:10.1056/NEJMoa0802656

6. Machiels J-PH, Haddad RI, Fayette J, et al. Afatinib versus methotrexate as second-line treatment in patients with recurrent or metastatic squamous-cell carcinoma of the head and neck progressing on or after platinum-based therapy (LUX-Head \& Neck 1): an openlabel, randomised Phase 3 trial. Lancet Oncol. 2015;16(5):583-594. doi:10.1016/S1470-2045(15)70124-5

7. Seiwert TY, Burtness B, Mehra R, et al. Safety and clinical activity of pembrolizumab for treatment of recurrent or metastatic squamous cell carcinoma of the head and neck (KEYNOTE-012): an open-label, multicentre, Phase 1b trial. Lancet Oncol. 2016;17(7):956-965. doi:10.1016/S1470-2045(16)30066-3

8. Ferris RL, Blumenschein G, Fayette J, et al. Nivolumab for recurrent squamous-cell carcinoma of the head and neck. $N$ Engl $J$ Med. 2016;375(19):1856-1867. doi:10.1056/NEJMoa1602252

9. Cohen EEW, Soulières D, Le Tourneau C, et al. Pembrolizumab versus methotrexate, docetaxel, or cetuximab for recurrent or metastatic head-and-neck squamous cell carcinoma (KEYNOTE-040): a randomised, open-label, phase 3 study. Lancet. 2019;393 (10167):156-167. doi:10.1016/S0140-6736(18)31999-8

10. O'Sullivan B, Huang SH, Su J, et al. Development and validation of a staging system for HPV-related oropharyngeal cancer by the international collaboration on oropharyngeal cancer network for staging (ICON-S): a multicentre cohort study. Lancet Oncol. 2016;17 (4):440-451. doi:10.1016/S1470-2045(15)00560-4

11. Mahal BA, Catalano PJ, Haddad RI, et al. Incidence and demographic burden of HPV-associated oropharyngeal head and neck cancers in the United States. Cancer Epidemiol Biomarkers Prev. 2019;28(10):1660-1667. doi:10.1158/1055-9965.EPI-19-0038

12. Kreimer AR, Ferreiro-Iglesias A, Nygard M, et al. Timing of HPV16-E6 antibody seroconversion before OPSCC: findings from the HPVC3 consortium. Ann Oncol. 2019;30(8):1335-1343. doi:10.1093/annonc/mdz138

13. Gillison ML, Broutian T, Pickard RK, et al. Prevalence of oral HPV infection in the United States, 2009-2010. JAMA. 2012;307 (7):693-703. doi:10.1001/jama.2012.101 
14. Sturgis EM, Ang KK. The epidemic of HPV-associated oropharyngeal cancer is here: is it time to change our treatment paradigms? $J$ Natl Compr Canc Netw. 2011;9(6):665-673. doi:10.6004/jncen. 2011.0055

15. Pinatti LM, Walline HM, Carey TE. Human papillomavirus genome integration and head and neck cancer. J Dent Res. 2018;97 (6):691-700. doi:10.1177/0022034517744213

16. Puram SV, Rocco JW. Molecular aspects of head and neck cancer therapy. Hematol Oncol Clin North Am. 2015;29(6):971-992. doi:10.1016/j.hoc.2015.07.003

17. Kanodia S, Fahey LM, Kast WM. Mechanisms used by human papillomaviruses to escape the host immune response. Curr Cancer Drug Targets. 2007;7(1):79-89. doi:10.2174/156800907780006869

18. Shi W, Kato H, Perez-Ordonez B, et al. Comparative prognostic value of HPV16 E6 mRNA compared with in situ hybridization for human oropharyngeal squamous carcinoma. J Clin Oncol. 2009;27 (36):6213-6221. doi:10.1200/JCO.2009.23.1670

19. Network CGA. Comprehensive genomic characterization of head and neck squamous cell carcinomas. Nature. 2015;517(7536):576-582. doi:10.1038/nature14129

20. Kang H, Kiess A, Chung CH. Emerging biomarkers in head and neck cancer in the era of genomics. Nat Rev Clin Oncol. 2015;12 (1):11-26. doi:10.1038/nrclinonc.2014.192

21. Gipson BJ, Robbins HA, Fakhry C, D’Souza G. Sensitivity and specificity of oral HPV detection for HPV-positive head and neck cancer. Oral Oncol. 2018;77:52-56. doi:10.1016/j.oraloncology.20 17.12.008

22. Leemans CR, Snijders PJF, Brakenhoff RH. The molecular landscape of head and neck cancer. Nat Rev Cancer. 2018;18(5):269-282. doi:10.1038/nrc.2018.11

23. Fukusumi T, Califano JA. The NOTCH pathway in head and neck squamous cell carcinoma. J Dent Res. 2018;97(6):645-653. doi: $10.1177 / 0022034518760297$

24. Stransky N, Egloff AM, Tward AD, et al. The mutational landscape of head and neck squamous cell carcinoma. Science. 2011;333 (6046):1157-1160. doi:10.1126/science. 1208130

25. Lakshmanachetty S, Balaiya V, High WA, Koster MI. Loss of TP63 promotes the metastasis of head and neck squamous cell carcinoma by activating MAPK and STAT3 signaling. Mol Cancer Res. 2019;17 (6):1279-1293. doi:10.1158/1541-7786.MCR-18-1355

26. Hayes DN, Van Waes C, Seiwert TY. Genetic landscape of human papillomavirus-associated head and neck cancer and comparison to tobacco-related tumors. J Clin Oncol. 2015;33(29):3227-3234. doi:10.1200/JCO.2015.62.1086

27. Jung K, Kang H, Mehra R. Targeting phosphoinositide 3-kinase (PI3K) in head and neck squamous cell carcinoma (HNSCC). Cancers Head Neck. 2018;3(1):3. doi:10.1186/s41199-018-0030-Z

28. Wang AT, Smogorzewska A. SnapShot: fanconi anemia and associated proteins. Cell. 2015;160(1-2):354-354.e351. doi:10.1016/j.cell.2014. 12.031

29. Hitt R, Irigoyen A, Cortes-Funes H, et al. Phase II study of the combination of cetuximab and weekly paclitaxel in the first-line treatment of patients with recurrent and/or metastatic squamous cell carcinoma of head and neck. Ann Oncol. 2012;23(4):1016-1022. doi:10.1093/annonc/mdr367

30. Chow LQM, Haddad R, Gupta S, et al. Antitumor activity of pembrolizumab in biomarker-unselected patients with recurrent and/or metastatic head and neck squamous cell carcinoma: results from the phase Ib KEYNOTE-012 expansion cohort. J Clin Oncol. 2016;34 (32):3838-3845. doi:10.1200/JCO.2016.68.1478

31. Larkins E, Blumenthal GM, Yuan W, et al. FDA approval summary: pembrolizumab for the treatment of recurrent or metastatic head and neck squamous cell carcinoma with disease progression on or after platinum-containing chemotherapy. Oncologist. 2017;22(7):873-878. doi:10.1634/theoncologist.2016-0496
32. Mehra R, Seiwert TY, Gupta S, et al. Efficacy and safety of pembrolizumab in recurrent/metastatic head and neck squamous cell carcinoma: pooled analyses after long-term follow-up in KEYNOTE-012. Br J Cancer. 2018;119(2):153-159. doi:10.1038/ s41416-018-0131-9

33. Kulangara K, Hanks DA, Waldroup S, et al. Development of the combined positive score (CPS) for the evaluation of PD-L1 in solid tumors with the immunohistochemistry assay PD-L1 IHC 22C3 pharmDx. J Clin Oncol. 2017;35(15_suppl):e14589e14589. doi:10.1200/JCO.2017.35.15_suppl.e14589

34. Bauml J, Seiwert TY, Pfister DG, et al. Pembrolizumab for platinumand cetuximab-refractory head and neck cancer: results from a single-arm, phase II study. J Clin Oncol. 2017;35(14):1542-1549. doi:10.1200/JCO.2016.70.1524

35. Burtness B, Harrington KJ, Greil R, et al. Pembrolizumab alone or with chemotherapy versus cetuximab with chemotherapy for recurrent or metastatic squamous cell carcinoma of the head and neck (KEYNOTE-048): a randomised, open-label, phase 3 study. Lancet. 2019;394(10212):1915-1928. doi:10.1016/S0140-6736(19) 32591-7

36. Bonnefoy-Berard N, Besnard V, Morel P, et al. Second signal for T lymphocyte activation: multiple targets for pharmacological modulation. Dev Biol Stand. 1992;77:41-48.

37. Shergold AL, Millar R, Nibbs RJB. Understanding and overcoming the resistance of cancer to PD-1/PD-L1 blockade. Pharmacol Res. 2019;145:104258. doi:10.1016/j.phrs.2019.104258

38. He Y, Cao J, Zhao C, Li X, Zhou C, Hirsch FR. TIM-3, a promising target for cancer immunotherapy. Onco Targets Ther. 2018;11:7005-7009. doi:10.2147/OTT

39. Solomon B, Young RJ, Rischin D. Head and neck squamous cell carcinoma: genomics and emerging biomarkers for immunomodulatory cancer treatments. Semin Cancer Biol. 2018;52(Pt 2):228-240. doi:10.1016/j.semcancer.2018.01.008

40. Buchbinder EI, Desai A. CTLA-4 and PD-1 pathways: similarities, differences, and implications of their inhibition. Am J Clin Oncol. 2016;39(1):98-106. doi:10.1097/COC.0000000000000239

41. Kleffel S, Posch C, Barthel SR, et al. Melanoma cell-intrinsic PD-1 receptor functions promote tumor growth. Cell. 2015;162 (6):1242-1256. doi:10.1016/j.cell.2015.08.052

42. Fourcade J, Sun Z, Benallaoua M, et al. Upregulation of Tim-3 and PD-1 expression is associated with tumor antigen-specific CD8+ T cell dysfunction in melanoma patients. J Exp Med. 2010;207 (10):2175-2186. doi:10.1084/jem.20100637

43. Zak KM, Grudnik P, Magiera K, Dömling A, Dubin G, Holak TA. Structural biology of the immune checkpoint receptor PD-1 and its ligands PD-L1/PD-L2. Structure. 2017;25(8):1163-1174. doi:10.1016/ j.str.2017.06.011

44. Strome SE, Dong H, Tamura H, et al. B7-H1 blockade augments adoptive T-cell immunotherapy for squamous cell carcinoma. Cancer Res. 2003;63(19):6501-6505.

45. Motzer RJ, Escudier B, McDermott DF, et al. Nivolumab versus everolimus in advanced renal-cell carcinoma. $N$ Engl $\mathrm{J}$ Med. 2015;373(19):1803-1813. doi:10.1056/NEJMoa1510665

46. Hansen AR, Siu LL. PD-L1 testing in cancer: challenges in companion diagnostic development. JAMA Oncol. 2016;2(1):15-16. doi:10.1001/jamaoncol.2015.4685

47. Taube JM, Klein A, Brahmer JR, et al. Association of PD-1, PD-1 ligands, and other features of the tumor immune microenvironment with response to anti-PD-1 therapy. Clin Cancer Res. 2014;20 (19):5064-5074. doi:10.1158/1078-0432.CCR-13-3271

48. Rischin D, Harrington KJ, Greil R. Protocol-specified final analysis of the phase 3 KEYNOTE-048 trial of pembrolizumab (pembro) as first-line therapy for recurrent/metastatic head and neck squamous cell carcinoma (R/M HNSCC). $J$ Clin Oncol. 2019;37 (15_suppl):6000. doi:10.1200/JCO.2019.37.15_suppl.6000 
49. Zandberg DP, Algazi AP, Jimeno A, et al. Durvalumab for recurrent or metastatic head and neck squamous cell carcinoma: results from a single-arm, phase II study in patients with $\geq 25 \%$ tumour cell PD-L1 expression who have progressed on platinum-based chemotherapy. Eur J Cancer. 2019;107:142-152. doi:10.1016/j.ejca.2018.11.015

50. Rasmussen JH, Lelkaitis G, Håkansson K, et al. Intratumor heterogeneity of PD-L1 expression in head and neck squamous cell carcinoma. Br J Cancer. 2019;120(10):1003-1006. doi:10.1038/ s41416-019-0449-y

51. Fakhry C, Zhang Q, Nguyen-Tan PF, et al. Human papillomavirus and overall survival after progression of oropharyngeal squamous cell carcinoma. J Clin Oncol. 2014;32(30):3365-3373. doi:10.1200/ JCO.2014.55.1937

52. Argiris A, Li S, Ghebremichael M, et al. Prognostic significance of human papillomavirus in recurrent or metastatic head and neck cancer: an analysis of Eastern cooperative oncology group trials. Ann Oncol. 2014;25(7):1410-1416. doi:10.1093/annonc/mdu167

53. Vermorken JB, Psyrri A, Mesía R, et al. Impact of tumor HPV status on outcome in patients with recurrent and/or metastatic squamous cell carcinoma of the head and neck receiving chemotherapy with or without cetuximab: retrospective analysis of the phase III EXTREME trial. Ann Oncol. 2014;25(4):801-807. doi:10.1093/annonc/mdt574

54. de Ruiter EJ, Ooft ML, Devriese LA, Willems SM. The prognostic role of tumor infiltrating T-lymphocytes in squamous cell carcinoma of the head and neck: a systematic review and meta-analysis. Oncoimmunology. 2017;6(11):e1356148. doi:10.1080/2162402X.2017.1356148

55. Keck MK, Zuo Z, Khattri A, et al. Integrative analysis of head and neck cancer identifies two biologically distinct HPV and three non-HPV subtypes. Clin Cancer Res. 2015;21(4):870-881. doi:10.1158/1078-0432.CCR-14-2481

56. Harbison RA, Kubik M, Konnick EQ, et al. The mutational landscape of recurrent versus nonrecurrent human papillomavirus-related oropharyngeal cancer. JCI Insight. 2018;3(14). doi:10.1172/jci.insight.99327

57. Yarchoan M, Hopkins A, Jaffee EM. Tumor mutational burden and response rate to $\mathrm{PD}-1$ inhibition. $N$ Engl $J$ Med. 2017;377 (25):2500-2501. doi:10.1056/NEJMc1713444

58. Chan TA, Yarchoan M, Jaffee E, et al. Development of tumor mutation burden as an immunotherapy biomarker: utility for the oncology clinic. Ann Oncol. 2019;30(1):44-56. doi:10.1093/annonc/mdy495

59. Rizvi NA, Hellmann MD, Snyder A, et al. Cancer immunology. Mutational landscape determines sensitivity to PD-1 blockade in non-small cell lung cancer. Science. 2015;348(6230):124-128. doi:10.1126/science.aaa1348

60. Cristescu R, Mogg R, Ayers M, et al. Pan-tumor genomic biomarkers for PD-1 checkpoint blockade-based immunotherapy. Science. 2018;362(6411):eaar3593. doi:10.1126/science.aar3593

61. Seiwert TY, Haddad R, Bauml J, et al. Abstract LB-339: biomarkers predictive of response to pembrolizumab in head and neck cancer (HNSCC). Cancer Res. 2018;78(13 Supplement):LB-339-LB-339.

62. Shaverdian N, Lisberg AE, Bornazyan K, et al. Previous radiotherapy and the clinical activity and toxicity of pembrolizumab in the treatment of non-small-cell lung cancer: a secondary analysis of the KEYNOTE-001 phase 1 trial. Lancet Oncol. 2017;18(7):895-903. doi:10.1016/S1470-2045(17)30380-7

63. Hu ZI, McArthur HL, Ho AY. The abscopal effect of radiation therapy: what is it and how can we use it in breast cancer? Curr Breast Cancer Rep. 2017;9(1):45-51. doi:10.1007/s12609-017-0234-y

64. Dovedi SJ, Adlard AL, Lipowska-Bhalla G, et al. Acquired resistance to fractionated radiotherapy can be overcome by concurrent PD-L1 blockade. Cancer Res. 2014;74(19):5458-5468. doi:10.1158/00085472.CAN-14-1258

65. Schapira E, Hubbeling H, Yeap BY, et al. Improved overall survival and locoregional disease control with concurrent PD-1 pathway inhibitors and stereotactic radiosurgery for lung cancer patients with brain metastases. Int $J$ Radiat Oncol Biol Phys. 2018;101 (3):624-629. doi:10.1016/j.ijrobp.2018.02.175
66. Oweida A, Hararah MK, Phan A, et al. Resistance to radiotherapy and PD-L1 blockade is mediated by TIM-3 upregulation and regulatory T-cell infiltration. Clin Cancer Res. 2018;24(21):5368-5380. doi:10.1158/1078-0432.CCR-18-1038

67. Sun XS, Sire C, Tao Y, et al. A phase II randomized trial of pembrolizumab versus cetuximab, concomitant with radiotherapy (RT) in locally advanced (LA) squamous cell carcinoma of the head and neck (SCCHN): first results of the GORTEC 2015-01 "PembroRad" trial. J Clin Oncol. 2018;36(15_suppl):6018. doi:10.1200/JCO.2018.36.15_suppl.6018

68. Ferris RL, Gillison ML, Harris J, et al. Safety evaluation of nivolumab (Nivo) concomitant with cetuximab-radiotherapy for intermediate (IR) and high-risk (HR) local-regionally advanced head and neck squamous cell carcinoma (HNSCC): RTOG 3504. J Clin Oncol. 2018;36(15_suppl):6010. doi:10.1200/JCO.2018.36.15_suppl.6010

69. Wise-Draper TM, Old MO, Worden FP, et al. Phase II multi-site investigation of neoadjuvant pembrolizumab and adjuvant concurrent radiation and pembrolizumab with or without cisplatin in resected head and neck squamous cell carcinoma. J Clin Oncol. 2018;36 (15_suppl):6017. doi:10.1200/JCO.2018.36.15_suppl.6017

70. Manukian G, Bar-Ad V, Lu B, Argiris A, Johnson JM. Combining radiation and immune checkpoint blockade in the treatment of head and neck squamous cell carcinoma. Front Oncol. 2019;9:122. doi:10.3389/fonc.2019.00122

71. Yu Y, Lee NY. JAVELIN head and neck 100: a phase III trial of avelumab and chemoradiation for locally advanced head and neck cancer. Future Oncol. 2019;15(7):687-694. doi:10.2217/fon-2018-0405

72. Siu LL, Licitra L, Tao YG, et al. Abstract CT163: KEYNOTE-412: pembrolizumab plus chemoradiation vs chemoradiation alone for locally advanced head and neck squamous cell carcinoma. Cancer Res. 2018;78(13 Supplement):CT163-CT163.

73. Uppaluri R, Lee NY, Westra W, et al. KEYNOTE-689: phase 3 study of adjuvant and neoadjuvant pembrolizumab combined with standard of care (SOC) in patients with resectable, locally advanced head and neck squamous cell carcinoma. J Clin Oncol. 2019;37(15_suppl): TPS6090-TPS6090. doi:10.1200/JCO.2019.37.15_supp1.TPS6090

74. Das S, Johnson DB. Immune-related adverse events and anti-tumor efficacy of immune checkpoint inhibitors. J Immunother Cancer. 2019;7(1):306. doi:10.1186/s40425-019-0805-8

75. Xing $P$, Zhang $F$, Wang $G$, et al. Incidence rates of immune-related adverse events and their correlation with response in advanced solid tumours treated with NIVO or NIVO+IPI: a systematic review and meta-analysis. J Immunother Cancer. 2019;7(1):341. doi:10.1186/ s40425-019-0779-6

76. Saleh K, Khalife-Saleh N, Kourie HR. Do immune-related adverse events correlate with response to immune checkpoint inhibitors? Immunotherapy. 2019;11(4):257-259. doi:10.2217/imt-2018-0201

77. Connolly C, Bambhania K, Naidoo J. Immune-related adverse events: a case-based approach. Front Oncol. 2019;9:530. doi:10.3389/fonc. 2019.00530

78. Läubli H, Koelzer VH, Matter MS, et al. The $\mathrm{T}$ cell repertoire in tumors overlaps with pulmonary inflammatory lesions in patients treated with checkpoint inhibitors. Oncoimmunology. 2018;7(2): e1386362. doi:10.1080/2162402X.2017.1386362

79. Khan Z, Hammer C, Guardino E, Chandler GS, Albert ML. Mechanisms of immune-related adverse events associated with immune checkpoint blockade: using germline genetics to develop a personalized approach. Genome Med. 2019;11(1):39. doi:10.1186/ s13073-019-0652-8

80. Anderson R, Theron AJ, Rapoport BL. Immunopathogenesis of immune checkpoint inhibitor-related adverse events: roles of the intestinal microbiome and Th17 cells. Front Immunol. 2019;10:2254. doi:10.3389/fimmu.2019.02254

81. Pierrard J, Seront E. Impact of the gut microbiome on immune checkpoint inhibitor efficacy-a systematic review. Curr Oncol. 2019;26(6):395-403. doi:10.3747/co.26.5177 
82. Tsao MS, Kerr KM, Kockx M, et al. PD-L1 immunohistochemistry comparability study in real-life clinical samples: results of blueprin phase 2 project. J Thorac Oncol. 2018;13(9):1302-1311. doi:10.1016/ j.jtho.2018.05.013

83. Segal NH, Ou SI, Balmanoukian A, et al. Safety and efficacy of durvalumab in patients with head and neck squamous cell carcinoma: results from a phase I/II expansion cohort. Eur $J$ Cancer. 2019;109:154-161. doi:10.1016/j.ejca.2018.12.029

84. Siu LL, Even C, Mesía R, et al. Safety and efficacy of durvalumab with or without tremelimumab in patients with $\mathrm{PD}-\mathrm{L} 1-\mathrm{low} /$ negative recurrent or metastatic HNSCC: the phase 2 CONDOR randomized clinical trial. JAMA Oncol. 2019;5(2):195-203. doi:10.1001/jamaoncol.2018.4628
85. Licitra LF, Haddad RI, Even C, et al. EAGLE: a phase 3, randomized, open-label study of durvalumab (D) with or without tremelimumab (T) in patients (pts) with recurrent or metastatic head and neck squamous cell carcinoma (R/M HNSCC). J Clin Oncol. 2019;37 (15_suppl):6012. doi:10.1200/JCO.2019.37.15_suppl.6012

86. Colevas AD, Bahleda R, Braiteh F, et al. Safety and clinical activity of atezolizumab in head and neck cancer: results from a phase I trial. Ann Oncol. 2018;29(11):2247-2253. doi:10.1093/annonc/mdy411

\section{Publish your work in this journal}

OncoTargets and Therapy is an international, peer-reviewed, open access journal focusing on the pathological basis of all cancers, potential targets for therapy and treatment protocols employed to improve the management of cancer patients. The journal also focuses on the impact of management programs and new therapeutic

Submit your manuscript here: https://www.dovepress.com/oncotargets-and-therapy-journal agents and protocols on patient perspectives such as quality of life, adherence and satisfaction. The manuscript management system is completely online and includes a very quick and fair peer-review system, which is all easy to use. Visit http://www.dovepress.com/ testimonials.php to read real quotes from published authors. 